\title{
Flexor Pollicis Longus Rupture in a Trigger Thumb after Intrasheath Triamcinolone Injections: A Case Report with Literature Review
}

\author{
Mitsuhiko Nanno', Takuya Sawaizumi², Norie Kodera ${ }^{2}$, \\ Yuji Tomori $^{2}$ and Shinro Takai ${ }^{2}$ \\ ${ }^{1}$ Department of Orthopaedic Surgery, Nippon Medical School Musashi Kosugi Hospital \\ ${ }^{2}$ Department of Orthopaedic Surgery, Nippon Medical School
}

\begin{abstract}
We report an unusual case of a rupture of the flexor pollicis longus tendon after two intrasheath triamcinolone injections for left trigger thumb in a 56-year-old woman. Tendon grafting from proximal to the wrist to the fingertip was carried out using the palmaris longus tendon. The clinical result was evaluated as good according to Strickland's criteria. Recently, intrasheath triamcinolone injection has been thought of as a mainstay of the treatment for trigger digits. However, flexor tendon ruptures have been reported as a serious, rare complication following the injections suspected to be the result of intratendinous injection. To avoid this complication, it is considered important to perform correct injection into the tendon sheath. Moreover, it is recommended that the number of injections should be once or twice, that a dose of triamcinolone should be less than $10 \mathrm{mg}$, and that, for preventing the flexor tendon rupture, intervals between injections should be more than three months. Given the potential for tendon rupture, surgeons should certainly recognize and alert patients to the risk of tendon ruptures even after only a few steroid injections.
\end{abstract}

(J Nippon Med Sch 2014; 81: 269-275)

Key words: flexor pollicis longus, tendon rupture, trigger thumb, intrasheath injections, triamcinolone

\section{Introduction}

Stenosing tenosynovitis of the digital flexor tendons or trigger finger is a well-known disease and one of the most common causes of hand pain and disability ${ }^{1.2}$. Generally, conservative treatment, such as limitation of the use of the disordered digits, splinting, using an anti-inflammatory analgesic ointment, or intrasheath corticosteroid injections, is often first recommended as the treatment of this disease $^{1-3}$. Especially, an intrasheath injection of triamcinolone acetonide has been proven to be an effective method for the treatment of it and has shown favorable results ${ }^{1-3}$. On the other hand, there have been reports of complications from the intrasheath injection technique, such as pain at the injection site, stiffness, depigmentation of the skin and subcutaneous fat atrophy, although those were rare, mild and self-limiting ${ }^{1.3}$. Moreover, a serious

Correspondence to Mitsuhiko Nanno, Department of Orthopaedic Surgery, Nippon Medical School Musashi Kosugi Hospital, 1-396 Kosugi-cho, Nakahara-ku, Kawasaki, Kanagawa 211-8533, Japan

E-mail: nanno-mi@ga2.so-net.ne.jp

Journal Website (http://www.nms.ac.jp/jnms/) 
potential complication of this treatment modality is a tendon rupture ${ }^{8-10}$. However, the flexor tendon rupture after the intrasheath corticosteroid injection is very rare. There are three case reports in the English literature ${ }^{4-6}$, and five in the Japanese literature $^{7-11}$. We present the current case of the flexor pollicis longus rupture in a trigger thumb after intrasheath triamcinolone injections, and discuss the diagnosis and treatment of this injury with reference to related studies.

\section{Case Peport}

A 56-year-old left-handed dominant woman presented with pain, swelling, and loss of flexion of her left thumb. Three years earlier, she had developed a left trigger thumb and was treated with an intrasheath injection $(1 \mathrm{~mL}$ local anesthetic and 1 $\mathrm{mL}(10 \mathrm{mg})$ triamcinolone acetonide [Kenacort-A 50 mg/5 mL, Bristol-Meyers Squibb Co., Princeton, NJ]) at another hospital. Her pain and triggering of the thumb was largely relieved after this injection. However, her symptoms recurred later. A second injection $(1 \mathrm{~mL}$ local anesthetic and $1 \mathrm{~mL}$ triamcinolone acetonide) was given at the same hospital soon after. Nevertheless no good relief of

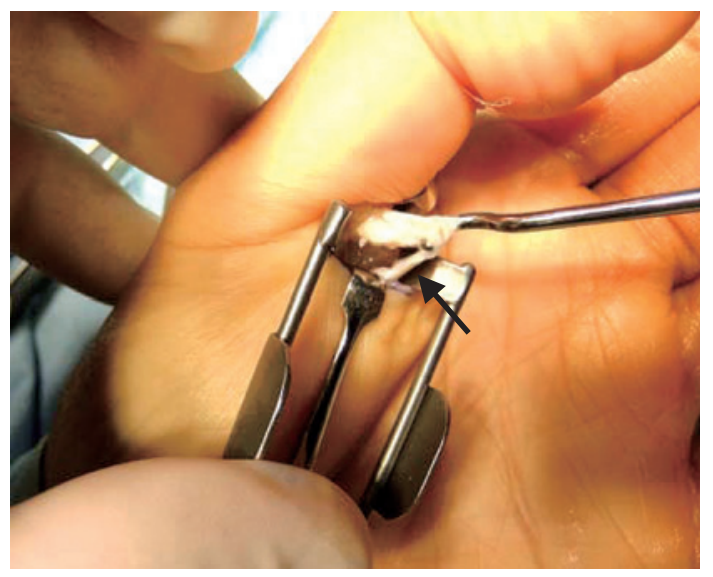

Fig. 1 Intraoperative photograph showing a partial rupture of the flexor pollicis longus (FPL) tendon at an another hospital before our first medical examination

Black solid arrow: a partial rupture of the FPL tendon

Surgical exposure shows that the FPL tendon was ruptured partially after the release of the tendon A1 pulley. symptoms was provided. She had persistent pain and tenderness over the A1 pulley. Therefore, she underwent operative release of the tendon A1 pulley under local anesthesia one month after the second injection. Although the flexor pollicis longus (FPL) was ruptured partially at operation, tendon reconstraction was not performed (Fig. 1). About one month after the surgery, while trying to put on her shoes, she felt a painful pop in her left thumb accompanied by complete loss of flexion of the interphalangeal joint (IP) and was referred to our hospital. On our first medical examination, she had tenderness and swelling on the palmar aspect of the metacarpophalangeal (MP) joint of the left thumb and was entirely unable to flex the IP joint (Fig. 2). There was no infection sign that indicated the purulent flexor tenosynovitis. She was in good health and had no history of rheumatoid arthritis, diabetes mellitus, collagen disease, wrist trauma, or systemic steroid use. Ultrasonography showed the distal end of the FPL rupture at the level of the MP joint, although the proximal end was not identified. Initial three-dimensional computed tomography (3D-CT), which can demonstrate the flexor tendon with the volume rendering technique, revealed the complete FPL tendon rupture and the defect of the tendon from the level of the MP joint to the carpal tunnel

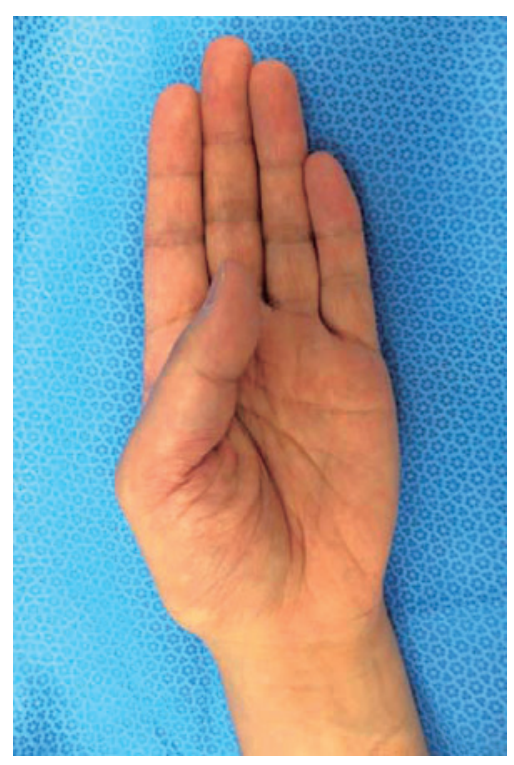

Fig. 2 Our first medical examination Physical examination revealed that the left thumb was unable to flex the interphalangeal joint (IP) entirely. 


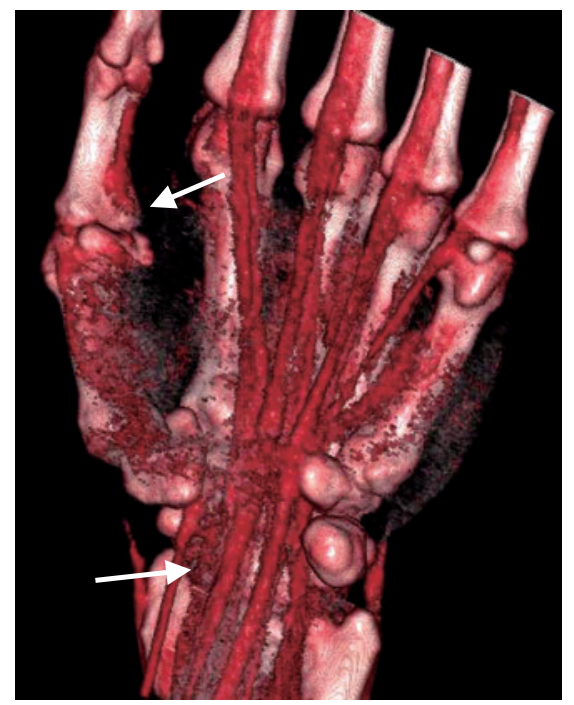

Fig. 3 3D-CT imaging with the volume rendering technique of left hand: posteroanterior view White solid arrows: both of the ruptured FPL tendon ends

3D-CT demonstrates the complete FPL tendon rupture and the defect of the tendon from the level of the metacarpophalangeal (MP) joint to the carpal tunnel.

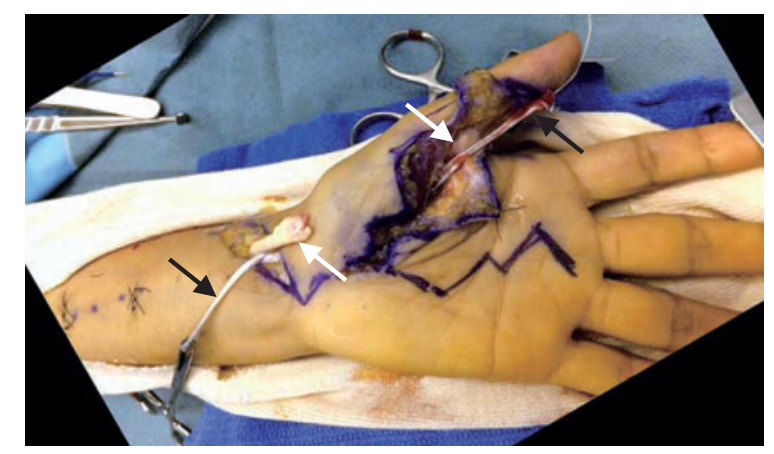

Fig. 4 Intraoperative photograph showing a ruptured FDP tendon and a grafted palmaris longus (PL)

(a) White solid arrows: both of the ends of the ruptured FPL tendon,

(b) Black solid arrows: a grafted PL

Surgical exposure shows that the FPL tendon was ruptured completely and the distance between the tendon ends was approximately $7 \mathrm{~cm}$, the same as the 3D-CT showed. Tendon grafting from proximal to the wrist to the fingertip was carried out using the PL tendon.

(Fig. 3). The laboratory results revealed no abnormal findings. A rupture of the FPL was suspected. One month after injury, surgical exploration confirmed

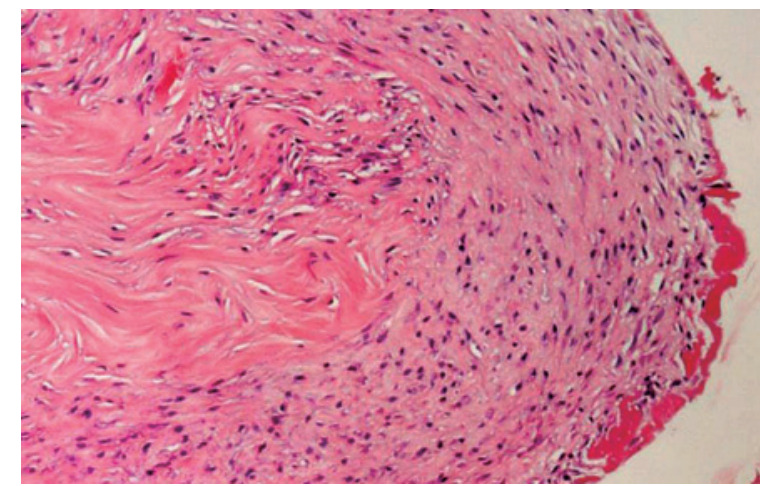

Fig. 5 Histological appearance of the biopsy of the ruptured FPL tendon end (hematoxylin and eosin stain $\times 100$ ).

The section reveals the presence of decreased viable fibroblast around degenerated tendon fiber with partial vascularization and slight inflammation.

that the FPL tendon was ruptured completely and its ends were identified, just as the 3D-CT showed. As the distance between the tendon ends was approximately $7 \mathrm{~cm}$ and direct suturing was not possible, tendon grafting from proximal to the wrist to the fingertip was carried out using the palmaris longus tendon (Fig. 4). Histologic examination of the ruptured tendon ends revealed the presence of decreased viable fibroblast around the degenerated tendon fiber with partial vascularization and slight inflammation (Fig. 5). The postoperative course was uneventful. Early mobilization in MP joint 90 degrees flexion with a dorsal splint was performed without fixing the IP joint so as to allow active movement immediately after surgery. The splint was removed at three weeks. At eighteen months follow-up evaluation, she no longer had any symptom. The range of motion of the affected thumb was $5^{\circ}$ to $55^{\circ}$ at the IP joint, and $0^{\circ}$ to $60^{\circ}$ at the MP joint. Percent total active motion (TAM) of the thumb was $63 \%$ (affected TAM 50\% unaffected TAM $\left.80^{\circ} \times 100\right)($ Fig. 6). According to Strickland's evaluation, the clinical result was evaluated as good.

\section{Discussion}

The trigger finger or stenosing tenosynovitis of the digit is one of the most common conditions of hand pain and dysfunction in the clinical practice of 
hand surgery ${ }^{1.2}$. Most primary trigger fingers can be successfully treated non-surgically, by limitation of the use of the disordered digits, application of a splint, or intrasheath corticosteroid injections ${ }^{1-3}$. Especially, corticosteroid injection, a pillar of nonsurgical management of trigger digit, is well described $^{1-3}$. Many authors have shown favorable results on intrasheath injection of betamethasone or triamcinolone ${ }^{1-3}$. In contrast, the side effects of the intrasheath injections, including subcutaneous fat atrophy, pain at the injection site, stiffness, and depigmentation of the skin, have been reported,

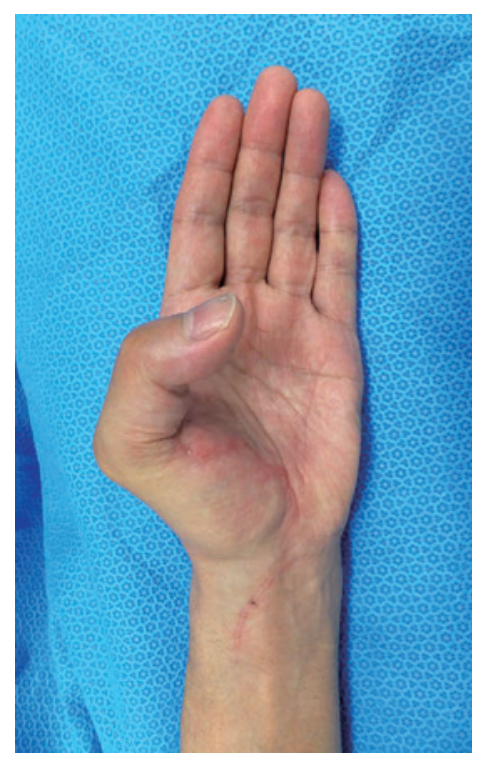

Fig. 6 The medical examination one year after the grafted PL tendon

Physical examination shows the range of motion of the thumb was $55^{\circ}$ flexion at the IP joint and $60^{\circ}$ flexion at the MP joint. although those complications were rare, generally mild and self-limiting ${ }^{1.3}$. However, more serious rare complications include tendon rupture or tendonsheath rupture ${ }^{4-6}$. There have been only three English reports and five Japanese reports on flexor tendon rupture after local corticosteroid injections for the treatment of a triggering digit, following suspected intratendinous injection ${ }^{4-11}$ (Table 1). Taras et al. first reported a case of rupture of the right flexor pollicis longus tendon in a 62-year-old patient with trigger thumb four years after two corticosteroid injections. This patient had a primary repair that failed by the re-rupture of the tendon and was ultimately treated with a tendon transfer ${ }^{4}$. Fitzgerald et al. then reported a case of rupture of the right middle FDP and FDS tendons in a 77-yearold patient with trigger finger thirteen months after two corticosteroid injections (triamcinolone acetonide $20 \mathrm{mg}$ ). This patient received a placement of a silicone rod as a tendon spacer, but finally opted to forgo the second stage of tendon reconstruction ${ }^{5}$. Recently, Yamada et al. reported a case of rupture of the right little FDP and FDS tendons in a 37-yearold patient with trigger finger two months after seven corticosteroid injections (triamcinolone acetonide $20 \mathrm{mg}$ ) over a fourteen month period. This patient had a tendon graft with the palmaris longus that failed by the rupture of the grafted tendon and was ultimately treated with a tendon re-graft using the opposite palmaris longus ${ }^{6}$.

Tendon ruptures after local corticosteroid injections in other anatomic parts, such as around

Table 1 Previous case reports of the FPL tendon rupture after intrasheath triamcinolon injections

\begin{tabular}{|c|c|c|c|c|c|c|}
\hline Author & Year & Finger & $\begin{array}{l}\text { Times of } \\
\text { injection }\end{array}$ & $\begin{array}{l}\text { Period of } \\
\text { injections }\end{array}$ & $\begin{array}{l}\text { Period between } \\
\text { last injection and } \\
\text { tendon rupture }\end{array}$ & $\begin{array}{c}\text { Dose of } \\
\text { triamcinolon }\end{array}$ \\
\hline Taras et al. & 1995 & Thumb & 2 & Unknown & $4 \mathrm{Y}$ & Unknown \\
\hline Fitzgerald et al. & 2005 & Middle & 2 & Unknown & $11 \mathrm{M}$ & $20 \mathrm{mg}$ \\
\hline Ishigami et al. & 2007 & Thumb & Unknown & $3 \mathrm{M}$ & Unknown & Unknown \\
\hline Sumiura et al.* & 2010 & Little & 3 & Unknown & $11 \mathrm{M}$ & Unknown \\
\hline Shimura et al.* & 2010 & Thumb & 5 & $3 \mathrm{Y}$ & Unknown & $20 \mathrm{mg}$ \\
\hline Yamada et al. & 2011 & Little & 7 & $14 \mathrm{M}$ & $2 \mathrm{M}$ & $20 \mathrm{mg}$ \\
\hline Hayami et al.* & 2011 & Thumb & 25 & $2 \mathrm{Y}$ & $\begin{array}{l}\text { Immediately } \\
\text { after injection }\end{array}$ & Unknown \\
\hline Tsubokawa et al.* & 2011 & Little & 2 & $2 \mathrm{~W}$ & $1 \mathrm{Y}$ & $20 \mathrm{mg}$ \\
\hline Current case & 2013 & Thumb & 2 & $3 \mathrm{Y}$ & $3 \mathrm{M}$ & $10 \mathrm{mg}$ \\
\hline
\end{tabular}

*: proceeding 
the knee, ankle, elbow, and shoulder also have been well described and characteristically occur when frequent injections have been given over a relatively short period ${ }^{12}$. Many in vitro and animal studies also have been reported in attempts to examine the relationship between corticosteroid injection and tendon rupture ${ }^{13,14}$. Some authors have, with animals, demonstrated that the intratendinous injection of corticosteroids results in collagen necrosis at the site of the injection, followed by a decrease in tensile strength, and eventual tendon ruptures ${ }^{13,14}$. In addition, a number of studies have evaluated effects of glucocorticoids at the cellular level. Tsai et al. reported that dexamethasone inhibits both the proliferation and the migration of cultured rat Achilles tendon fibroblasts ${ }^{15}$. Wong et al. reported that glucocorticoid significantly suppressed human tenocyte cell viability, cell proliferation, proteoglycan production, and its collagen synthesis on cultured healthy patellar tendon ${ }^{16}$. And they suggest that disturbed tenocyte function may affect the structural integrity and the viscoelastic properties of a tendon and predispose it to subsequent spontaneous rupture ${ }^{16}$. All these reports suggest that intratendinous steroid injection should be avoided when injecting for tendinitis, because of the deleterious effects of steroids on the tenocyte ${ }^{1.9}$.

The standard technique practised for local corticosteroid injection is well related ${ }^{1,2,5,14}$. The corticosteroid injection is performed at the level of the distal palmar crease just proximal to the A1 pulley $^{1,2,5,14}$. A 25- or 27-gauge needle is vertically inserted into the flexor tendon and slight pressure is put on the plunger as the needle is being pulled gradually. Once a remarkable decrease in resistance is experienced, the remainder of the corticosteroid is injected to avoid the risk of intratendinous injection, attempting to fill the tendon sheath. Taras et al. are careful not to attempt injection when significant resistance is encountered, considering that such resistance may show the probability of intratendinous injection ${ }^{4}$. However, trying the intrasheath injection poses risk of injecting into the substance of the tendon. Consequently, the injection may increase the negative effects on collagen synthesis and ultimately lead to tendon rupture ${ }^{5}$. To improve treatment results of corticosteroid injection, it is considered important to perform accurate injection into the tendon sheath ${ }^{1}$. Fitzgerald et al. recommend moving the finger gently, actively or passively, to check whether the inserted needle is intratendinous or not, after needle placement into the tendon ${ }^{5}$. This causes slight motion of the needle, which is withdrawn gradually until no further motion is produced despite continued mild finger motion. The steroids then should be injected making sure that the injection be intrasheath. In the present circumstances, there is a limitation of the technique to inject steroid into the tendon sheath after inserting into the tendon percutaneously using a needle. Sawaizumi et al. ${ }^{1}$ reported no complications caused by using a 26- or 27-gauge needle. To reduce the possibility of invasion of the tendon, it is currently considered to be desirable to use a fine needle, such as a 26 - or 27-gauge needle.

The suitable numbers of corticosteroid injections, dose and intervals between injections for treating a trigger digit, have not yet been determined, though some authors advise a series of up to two corticosteroid injections over the course of three weeks ${ }^{5,14}$. Reviewing previous eight case reports of the flexor tendon rupture after intrasheath triamcinolone injections, we may summarize that the number of injections was more than twice, the period between the last injection and tendon rupture was more than eleven months, and the dose of triamcinolone was $20 \mathrm{mg}$ in all cases ${ }^{4-11}$ (Table 1). Sarris et al. $^{14}$ reported that in one series $93 \%$ of patients with trigger digits treated with one injection remained symptom free for three months. Therefore, we recommend that the number of injections should be once or twice, that the dose of triamcinolone is less than $10 \mathrm{mg}$, and that intervals between injections is more than three months for preventing the flexor tendon rupture, as Sawaizumi et al. ${ }^{1}$ stated. The flexor tendon rupture is generally thought of as having very low morbidity ${ }^{4-6}$. Given the potential for tendon rupture, however, surgeons should certainly recognize and alert patients to the risk of tendon ruptures after only a few steroid injections.

As for the diagnosis of flexor tendon rupture, 
preoperative identification of a ruptured tendon stump site may be useful in planning of the surgical approach and method. Ultrasound is a valuable diagnostic method in tendon injuries, and a noninvasive, dynamic examination, and does not irradiate the patient. However, the accuracy of the diagnosis by ultrasound is very much dependent upon the examiner. Moreover, it sometimes is difficult to reveal the end of tendon ruptures with a long tendon defect. On the other hand, 3D-CT with the volume rendering technique can demonstrate the relationship between bone structure and flexor tendons simultaneously in the hand and wrist ${ }^{17}$. In the current case, the FPL rupture was also obviously depicted by this 3D-CT imaging. And it could be helpful for the diagnosis and the selection of surgical method.

Treatment options for reconstruction of FPL rupture include direct repair, tendon graft, and tendon transfer. Taras et al. reported that the primary direct repair failed, in spite of the fact that the tendon stock appeared to be acceptably healthy after the worn-out ends of the rupture were debrided $^{4}$. They suggested that the intrinsic healing capacity of the tendon at the site of rupture was reduced with failure occurring late after direct repair. ${ }^{4}$ One standard treatment for this tendon rupture has been tendon grafting from proximal to the wrist to the fingertip carried out using the palmaris longus tendon. Moreover, the tendon graft using the palmaris longus is more physiological than the tendon transfer from the flexor digitorium superficialis of the middle finger or the ring finger, and can be performed without loss of finger flexor power. Therefore, we consider that the tendon graft is a good option for reconstruction of a ruptured tendon after intrasheath corticosteroid injection. However, Yamada et al. reported that a case of a tendon graft with the palmaris longus that failed by the rupture of the grafted tendon was ultimately treated with a tendon re-graft using the opposite palmaris longus ${ }^{6}$. Conclusively, it is recommended that both of the treatments should be given: the sufficient debridement of the bilateral ends of the ruptured tendon that the intrinsic healing capacity of which was reduced by triamcinolone and tendon graft using the palmaris longus tendon as well as careful early mobilization are performed for this injury.

Conflict of Interest: The authors declare no conflict of interest.

\section{References}

1. Sawaizumi T, Nanno M, Ito $\mathrm{H}$ : Intrasheath triamcinolone injection for the treatment of trigger digits in adult. Hand Surg 2005; 10: 37-42.

2. Wolfe SW: Tendinopathy. In Green's Operative Hand Surgery (Green DP, Hotchkiss RN, Pederson WC, eds), 6th ed. Vol. 2. 2011; pp 2067-2079, Churchill Livingstone, New York.

3. Freiberg A, Mulholland RS, Levine R: Nonoperative treatment of trigger fingers and thumbs. J Hand Surg 1989; 14A: 553-558.

4. Taras JS, Iiams GJ, Gibbons M, Culp RW: Flexor pollicis longus rupture in a trigger thumb: a case report. J Hand Surg 1995; 20A: 276-277.

5. Fitzgerald BT, Hofmeister ER, Fan RA, Thompson MA: Delayed flexor digitorum superficialis and profundus ruptures in a trigger finger after a steroid injection: a case report. J Hand Surg 2005; 30A: 479482.

6. Yamada K, Masuko T, Iwasaki N: Rupture of the flexor digitorum profundus tendon after injections of insoluble steroid for a tigger finger. J Hand Surg 2011; 15E: 77-78.

7. Ishigami S, Taniguchi Y, Tanihashi Y, Yoshida M: Delayed flexor pollicis longus rupture in a trigger thumb. Cent Jap J Orthop Traumat 2007; 50: 851852.

8. Sumiura S, Yamamoto M, Nagahiro Y, Ikeda Y, Muto M: A case of flexor digitorum profundus rupture in a trigger little finger after intrasheath triamcinolone injections. Seikeigeka to Saugaigeka 2010; 59 (Suppl 2) : 117.

9. Tsubokawa N, Maki Y, Narisawa H, Moriya K, Yoshizu T, Kusano N: A case of flexor digitorum profundus rupture of little finger and the pulley ruptures of middle and ring finger after intrasheath triamcinolone injections. J Jpn Soc Surg Hand 2011; 28: 119.

10. Hayami N, Shigematu K, Ohta D, Saito M, Ishizaki Y, Tanaka Y: A case of flexor pollicis longus rupture in a trigger thumb after several intrasheath triamcinolone injections. Cent Jap J Orthop Traumat 2011; 55 (Suppl 2) : 87.

11. Shimura H, Yamada T, Wakabayashi Y: A case of flexor pollicis longus rupture in a trigger thumb after intrasheath triamcinolone injections. J Jpn Soc Surg Hand 2011; 26: 493.

12. Ford LT, DeBender J: Tendon rupture after local steroid injection. South Med J 1979; 72: 827-830.

13. Fredburg U: Local corticosteroid injection in sport: review of literature and guidelines for treatment. Cand J Med Sci Sports 1997; 7: 131-139.

14. Sarris I, Darlis NA, Musgrave D, Sotereanos DG: 
Tenosynovitis: trigger finger, de Quervain Syndrome, flexor carpi radialis, and extensor carpi ulnaris. In Hand, Elbow and Shoulder: Core Knowledge In Orthopaedics (Trumble TE, Budoff JE, Coenwall R, eds), 2006; pp 212-221, Mosby, Philadelphia.

15. Tsai WC, Tang FT, Wong MK, Yen HC, Pang JH: Decreased expression of proliferating cell nuclear antigen is associated with dexamethasone inhibition of the proliferation of rat tendon fibroblasts. J Rheumatol 2002; 29: 2397-2402.

16. Wong MW, Tang YN, Fu SC, Lee KM, Chan KM Triamcinolone suppresses human tenocyte cellular activity and collagen synthesis. Clin Orthop Relat Res 2004; 421: 277-281.

17. Sunagawa T, Ochi M, Ishida O, Ono C, Ikuta Y: Three-dimensional CT imaging of flexor tendon ruptures in the hand and wrist. J Comput Assist Tomogr 2003; 27: 169-174.

(Received, August 1, 2013)

(Accepted, September 11, 2013) 